\title{
ALGĖBRES $A$-CONVEXES ET PROBLÈME DE MICHAEL
}

\author{
M. AKKAR, L. OUBBI, AND M. OUDADESS
}

(Communicated by Palle E. T. Jorgensen)

\begin{abstract}
We give an example of a locally $A$-convex algebra which is neither $m$-convex nor uniformly $A$-convex. This example is used to answer some questions on $A$-convex algebras (see symbol 4). We especially show that Michael's problem (in $m$-convex algebras) is equivalent to the same problem in $A$-convex algebras. Finally some questions on bounded sets in $A$-convex algebras are studied.
\end{abstract}

\section{INTRODUCTION}

Jusqu'ici, les seuls exemples connus d'algèbres localement $A$-convexes, non $m$-convexes ni uniformément localement $A$-convexes, sont des produits finis d'algèbres localement $m$-convexes et d'algèbres uniformément localement $A$ convexes. Ces exemples ont été construits pour la première fois en 1985 [6], mais ils présentent l'inconvenient de conserver, étant des produits finis, beaucoup de bonnes propriétés des algèbres à partir desquelles ils ont été construits. Nous donnons ici un exemple d'algèbre localement $A$-convexe qui n'est d'aucun de ces deux types et qui, a priori, n'est pas un produit. Cet exemple nous permet de répondre à certaines questions sur les algèbres localement $A$-convexes.

Dans [9], M. Oudadess munit toute algèbre localement $A$-convexe $(E, \tau)$ d'une topologie localement $m$-convexe $M(\tau)$ plus fine que $\tau$. Il montre que si $\tau$ est complete, il en est de même de $M(\tau)$ mais il ne dit rien sur la réciproque. Il ne dit rien non plus sur les bornés de $\tau$ et de $M(\tau)$. Notre exemple montre que la réciproque en question est fausse et que $\tau$ et $M(\tau)$ n'ont pas, en général, les mêmes bornés. Nous montrons, cependant, que sous différentes notions de complétudes $\tau$ et $M(\tau)$ ont les mêmes bornés d'un certain type. Ceci nous permet de montrer que le problème de savoir si tout caractère d'une algèbre localement $m$-convexe commutative et complète est borné [7], est équivalent au même problème dans les algèbres localement $A$-convexes. Il permet aussi de donner une réponse partielle à la question posée par Cochran dans[2, 3.6].

Received by the editors March 27, 1989; the contents of this paper have been presented at the meeting of the International Center of Pure and Applied Mathematics, August-September 1986, Nice, France.

1980 Mathematics Subject Classification (1985 Revision). Primary 46H10; Secondary 46H99.

Key words and phrases. Locally $A$-convex algebras, locally multiplicatively convex algebras, bounded sets, Michael Problem. 


\section{Preliminaires}

On désignera par $E$ une algèbre associative sur le corps $K(=\mathbb{R}$ ou $\mathbb{C})$ et par $\tau$ une topologie d'espace localement convexe (e.l.c) sur $E$.

On dira que $(E, \tau)$ est une algèbre localement convexe (a.l.c) si la multiplication de $E$ est séparément continue.

Une a.l.c $(E, \tau)$ est dite une algèbre localement $A$-convexe (a.l. $A$-c) si $\tau$ peut étre définie par une famille $\left(P_{\lambda}\right)_{\lambda}$ de seminormes $A$-convexes, i.e. telles que, pour tout $x$ de $E$ et tout $\lambda$, il existe $M(x, \lambda)$ et $N(x, \lambda)$ des réels positifs vérifiant:

$$
P_{\lambda}(x y) \leq M(x, \lambda) P_{\lambda}(y), \quad \forall y \in E
$$

et

$$
P_{\lambda}(y x) \leq N(x, \lambda) P_{\lambda}(y), \quad \forall y \in E .
$$

Si les constantes $M(x, \lambda)$ et $N(x, \lambda)$ peuvent étre prises égales à $P_{\lambda}(x)$ (resp. indépendantes de $\lambda$, i.e. $M(x, \lambda)=M(x)$ et $N(x, \lambda)=N(x))$ alors $(E, \tau)$ est dite une algèbre localement multiplicativement convexe (a.l.m.c) (resp. une algèbre uniformément localement $A$-convexe (a.u.l. $A$-c.)).

Un disque borné $B$ d'un e.l.c séparé $F$ est dit complétant si le sous-espace vectoriel $F_{B}$ de $F$ engendré par $B$ muni de la jauge de $B$ est un espace de Banach.

On dira qu'un e.l.c séparé $(F, T)$ est bornologiquement complet ( $b$-complet) si tout borné de $F$ est contenu dans un disque borné complétant.

Une a.l.c séparée est dite pseudo-complète si tout disque borné fermé et idempotent est complétant.

Un borné d'une a.l.c est régulier s'il est absorbé par un disque borné idempotent.

\section{UN EXEMPLE D'ALGĖBRE LOCALEMENT $A$-CONVEXE}

Soit $E=C(\mathbb{R})$ l'algèbre des fonctions complèxes continues sur $\mathbb{R}$. On définit sur $E$, pour tout $n$ dans $\mathbb{N}^{*}$, la semi-norme suivante: $P_{n}(f)=\int_{-n}^{n}|f(t)| d t$. Soit $\tau$ la topologie définie par les $P_{n}, n \in N^{*}$. Alors $(E, \tau)$ est clairement une a.l. $A$-c. Comme toute a.u.l. $A$-c est à éléments réguliers [8], $(E, \tau)$ n'est pas une a.u.l. $A$-c. En effet tout élément non borné de $E$ est non régulier. Par ailleurs, en utilisant une technique analogue à celle utilisée dans [3], on montre que $(E, \tau)$ n'est pas une a.l.m.c. Ainsi $(E, \tau)$ donne t-il un exemple d'a.l. $A$-c qui n'est ni $m$-convexe ni u.l. $A$-c ni, a priori, construit à partir de telles algèbres.

\section{Topologie D'A.L.M.C DANS LES A.L. $A$-C}

Soit $(E, \tau)$ une a.l. $A$-c unitaire. On suppose que $\tau$ est définie par la famille $\left(P_{\lambda}\right)_{\lambda \in \Lambda}$ de semi-normes $A$-convexes. On définit sur $E$ la semi-norme $q_{\lambda}$ par: $q_{\lambda}(x)=\sup \left\{p_{\lambda}(x y), p_{\lambda}(y) \leq 1\right\}$. Alors la famille $\left(q_{\lambda}\right)_{\lambda \in \Lambda}$ définit sur $E$ une topologie d'a.l.m.c $M(\tau)$ plus fine que $\tau$ [9]. Dans le cas non unitaire, on considère $\left(E_{1}, \tau_{1}\right)$ l'a.l. $A$-c obtenue par adjonction d'une unité à $(E, \tau)$, et l'on considère la topologie, notée enccre $M(\tau)$, induite sur $E$ par $M\left(\tau_{1}\right)$. 
Dans ce cas $M(\tau)$ n'est pas nécessairement définie par les $q_{\lambda}$. Pour voir ceci, il suffit de considerer une algèbre normée $E$ admettant un élément non nul $x$ tel que $x . E=\{0\}$ [1]. Cependant $M(\tau)$ admet toujours une base locale en $O$ formée de $\tau$-tonneaux. Ceci résulte du fait que la trace d'un $\tau_{1}$-tonneau de $E_{1}$ sur $E$ est un $\tau$-tonneau et que chaque $B_{\lambda}(\varepsilon)=\left\{x \in E: q_{\lambda}(x) \leq \varepsilon\right\}$ est un $\tau$-tonneau.

Remarque 4.1. Dans le cas de l'exemple précédent, la topologie $M(\tau)$ n'est autre que la topologie de la convergence uniforme sur les compacts de $\mathbb{R}$. Pour voir ceci, on passe aux algèbres quotients $C[-n, n]$ de $C(\mathbb{R})$ par $P_{n}^{-1}(0), n \geq$ 1. On munit chaque $C[-n, n]$ de la $A$-norme quotient $\bar{P}_{n}$ et on considère $\bar{q}_{n}$, la norme de Cochran [4] associée à $\bar{P}_{n}$. On remarque alors que $q_{n}(f)=\bar{q}_{n}(\bar{f})$, $f \in C(\mathbb{R})$. Or $\bar{P}_{n}$ est exactement la norme $L^{1}$ sur $C[-n, n]$. Donc $\bar{q}_{n}$ est la norme infinie sur $[-n, n]$.

Il est donc clair que dans ce cas $M(\tau)$ est complète alors que $\tau$ ne l'est pas; car sinon $\tau$ étant métrisable, elle coinciderait avec $M(\tau)$ ce qui n'est pas vrai. Ainsi la réponse à la question de savoir si $\tau$ est complete dès que $M(\tau)$ l'est est négative.

Ce même exemple montre, qu'en général $\tau$ et $M(\tau)$ n'ont pas les mêmes bornés. Encore une fois, étant métrisables, $\tau$ et $M(\tau)$ coincideraient si elles avaient les mêmes bornés.

\section{ENSEMBLES BORNÉS}

Nour donnons dans ce qui suit différentes conditions sous lesquelles $\tau$ et $M(\tau)$ ont les mêmes bornés d'un certain type. Pour ce faire on a besoin du lemme classique suivant:

Lemme 5.1. Dans un espace localement convexe séparé tout tonneau absorbe les disques bornés complétants.

Nous obtenons alors le résultat principal de ce papier.

Thèorème 5.2. Soit $(E, \tau)$ une a.l. $A$-c séparée.

(1) Si $(E, \tau)$ est pseudo-complete, alors $\tau$ et $M(\tau)$ ont les mêmes bornés réguliers.

(2) Si $(E, \tau)$ est b-complète, alors $\tau$ et $M(\tau)$ ont les mêmes bornés.

Preuve. Il est clair que tout $M(\tau)$-borné est $\tau$-borné. Il reste donc à montrer les implications inverses.

(1) $\mathrm{Si} B$ est un borné régulier de $(E, \tau)$, alors il est absorbé par un disque borné fermé et idempotent $D$. Or $(E, \tau)$ est pseudo-complete. Donc $D$ est completant; et comme tout $M(\tau)$-voisinage $V$ de $O$ contient un $\tau$-tonneau, $D$ est absorbé par $V$. Donc $B$ est aussi borné pour $M(\tau)$.

(2) On raisonne de même en remarquant que tout disque fermé borné d'un espace localement convexe séparé $b$-complet est complétant. 
Corollaire 5.3. Si $(E, \tau)$ est une a.l. A-c complète, alors $\tau$ et $M(\tau)$ ont les mêmes bornés.

On en arrive maintenant au problème de Michael.

Corollaire 5.4. Les assertions suivantes sont équivalentes:

(1) Tout caractère d'une a.l. A-c commutative complète est borné.

(2) Tout caractère d'une a.l.m.c commutative complète est borné.

La proposition suivante donne une autre condition sous laquelle $\tau$ et $M(\tau)$ ont les mêmes bornés.

Proposition 5.5. Soit $(E, \tau)$ une a.l. A-c séparée ayant pour dual $E^{\prime}$. Si la topologie de Machey $\tau\left(E, E^{\prime}\right)$ est tonnelée, alors $(E, M(\tau))$ admet aussi $E^{\prime}$ pour dual. En particulier $\tau$ et $M(\tau)$ ont les mêmes bornés.

Cette proposition permet de donner une réponse partielle à la question (3.6) de [2]. Avec les notations de [2] on a

Proposition 5.6. Si $\tau\left(E, E^{\prime}\right)$ est tonnelée, alors $\Sigma\left(E, E^{\prime}\right)$ et $x\left(E, E^{\prime}\right)$ coincident.

Preuve. Il suffit de montrer que $x\left(E, E^{\prime}\right)$ est plus fine que $\Sigma\left(E, E^{\prime}\right)$. L'autre sens est toujours vrai. On considère la topologie $M\left(\Sigma\left(E, E^{\prime}\right)\right)$. Par la proposition précédente celle-ci est compatible avec la dualité $\left(E, E^{\prime}\right)$. Donc elle est moins fine que $x\left(E, E^{\prime}\right)$. Par suite $x\left(E, E^{\prime}\right)=\Sigma\left(E, E^{\prime}\right)$.

Remarque 5.7. La complétude de $\tau$ ne suffit pas pour que $\tau$ et $M(\tau)$ soient compatibles avec la même dualité. Il suffit de considerer l'algèbre $\left(c_{b}(R), \beta\right)$ des fonctions continues bornées sur $\mathbb{R}$ munie de la topologie stricte. C'est une a.u.l. $A$-c de Mackey [5]. Donc $M(\beta)$ n'est pas compatible avec la même dualité que $\beta$.

La pseudo-complètude ne suffit pas dans le Théorème 5.2 pour avoir les mêmes bornés. Il suffit de considérer l'algèbre $\left(C[0,1],\|\cdot\|_{1}\right)$. C'est une algèbre $A$-normée pseudo-complète. Cependant les normes $\|\cdot\|_{1}$ et \|\|$_{\infty}$ ne sont pas équivalentes.

Pour terminer remarquons que les assertions suivantes sont équivalentes:

(i) $\tau$ et $M(\tau)$ ont les mêmes bornés.

(ii) $\forall B$ borné de $(E, \tau), \forall \lambda \in \Lambda, \exists M(B, \lambda)>0$ tel que

$$
P_{\lambda}(x y) \leq M(B, \lambda) \cdot P_{\lambda}(y) ; x \in B, y \in E .
$$

\section{RÉFÉRENCES}

1. O. H. Cheikh and M. Oudadess, On a commutativity question in Banach algebras, Arab. Gulf. J. Res. Math. Phys. Sci. A 6 (1988), 173-179.

2. A. C. Cochran, Topological algebras and Mackey topologies, Proc. Amer. Math. Soc. 30 (1973), 473-479.

3. __ Representation of A-convex algebras, Proc. Amer. Math. Soc. 41 (1973), 473-479. 
4. A. C. Cochran, R. Keown, and C. R. Williams, On a class of topological algebras, Pacific J. Math. 34 (1970), 17-25.

5. J. B. Conway, The strict topology and compactness in the space of measures, Bull. Amer. Math. Soc. 72 (1966), 75-78.

6. H. Kemmoun, Contribution aux algèbres localement A-convexes, Thèse de 3ème Cycle, Ėcole Normale Supérieure Takaddoum, Rabat, Maroc, 1986.

7. A. Michael, Locally multiplicatively convex topological algebras, Mem. Amer. Math. Soc., no. 11, Amer. Math. Soc., Providence, RI, 1952.

8. M. Oudadess, Théorèmes de structure et propriétés fondamentales des algèbres localement uniformément A-convexes, C.R. Acad. Sci. Paris Sér. I Math. 296 (1983), 851-853.

9. __ Fundamental properties of locally A-convex algebras, Pub. Math., École Normale Supérieure Takaddoum, No. 1, Rabat, Maroc., 1985-86, pp. 7.1-7.6.

École Normale Supérieure, TaKaddoum, B. P: 5118-Rabat-Maroc 